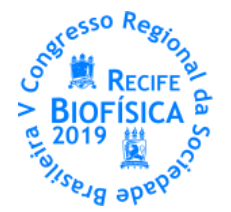

\title{
ALTERAÇÕES ELETROFISIOLÓGICAS NO POTENCIAL DE AÇÃO COMPOSTO DO NERVO CIÁTICO DE RATOS NO DIABETES MELLITUS INDUZIDO POR STZ NO PERÍODO ADULTO
}

Rutyleia Alves-Soares ${ }^{1 *}$, Lívia Carolina Amâncio², Yuri de Abreu Gomes-Vasconcelos ${ }^{1}$, Amauri Barbosa da SilvaJunior $^{1}$, Antonio Lucas Fernandes-Leal ${ }^{1}$, Maria Natália Feitosa-de-Sousa', Alan Hílame Diniz-Gomes ${ }^{1}$, Larissa BatistaBessa $^{1}$, Kerly Shamyra da Silva-Alves ${ }^{2}$, Francisco Walber Ferreira-da-Silva ${ }^{2}$, José Henrique Leal-Cardoso ${ }^{2}$, Andrelina Noronha Coelho-de-Souza ${ }^{1}$

1'Laboratório de Fisiologia Experimental, ISCB/UECE, ${ }^{2}$ Laboratório de Eletrofisiologia e Contração Muscular, ISCB/UECE *rutyleia.alves@gmail.com

\section{INTRODUÇÃO}

O Diabetes mellitus (DM) é uma doença metabólica crônica caracterizado por um quadro de hiperglicemia devido a falhas na produção ou sensibilidade a insulina, levando a danos em diversos órgãos e sistemas, inclusive no Sistema Nervoso (LIRK et al., 2015). A neuropatia diabética periférica (NDP) é a complicação mais comum do DM, levando à maior morbidade e mortalidade podendo ser vista como um problema de saúde pública. Fatores causais como hiperglicemia persistente, insuficiência microvascular, estresse oxidativo e nitrosativo, neurotropismo defeituoso e destruição nervosa autoimune, podem desencadear a NDP culminando em alterações na atividade elétrica de nervos. NDP pode atingir tanto fibras mielinizadas como não-mielinizadas (VINIK et al., 2013). Considerado como o maior nervo do corpo humano, o nervo ciático (NC) é o mais afetado pela NDP, causando danos motores e sensoriais nos membros inferiores (FREEMAN et al., 2016). Assim, objetivou-se avaliar as alterações eletrofisiológicas do NC em ratos induzidos a diabetes por STZ no período adulto, após 12 semanas da indução.

\section{MATERIAIS E MÉTODOS}

Foram utilizados ratos Wistar (Rattus novergicus) machos na $8^{\text {a }}$ semana de vida com peso entre $200 \pm 20 \mathrm{~g}$. Os animais foram fornecidos pelo Biotério do Centro Universitário Christus (UniChristus) e mantidos no Biotério da Universidade Estadual do Ceará (UECE), em caixas de polipropileno com livre acesso a água e ração. A pesquisa foi aprovada pelo Comitê de Ética para Uso de Animais (CEUA-UECE) sob código 0854496/2018. Para indução do $\mathrm{DM}$, os animais do grupo Diabético ( $\mathrm{DM}, \mathrm{n}=7$ ) receberam uma dose única de STZ $(65 \mathrm{mg} / \mathrm{kg}$ (i.p.). 0 grupo controle $(C T, n=8)$ recebeu apenas o veículo (Citrato de Sódio, 0,1 M, pH 4,5, i.p). Após a indução e confirmação do DM, os animais foram acompanhados por 12 semanas e para confirmação e caracterização do modelo, foram aferidos, semanalmente, parâmetros como: glicemia de jejum, peso, ingesta de água e ração e diurese. Ao término do acompanhamento, os animais foram eutanasiados e em seguida o NC foi dissecado desde as raízes nervosas até sua porção subplantar, nutrido com solução de Locke. O NC então, foi colocado em uma câmara de Harvard com solução nutridora de Locke, e estimulado da parte proximal para distal com estímulo de $40 \mathrm{~V}$, com duração de $0,1 \mathrm{~ms}$, a uma frequência de $0,2 \mathrm{~Hz}$ (GRASS S48, Grass Instruments), para estimulação de fibras mielinizadas. $\mathrm{O}$
Potencial de Ação Composto (PAC), foi então coletado, amplificado e enviado para o osciloscópio (TDS 340A, Tektronix). Por meio da placa de interface analógico/digital (DIGIDATA 1322 A, Axon Instruments), o sinal foi finalmente enviado para um computador equipado com o software Axoclamp ${ }^{\circledR}$ (p-clamp 10, Molecular Devices) para armazenamento e posterior análise do sinal. Os resultados foram expressos por média $\pm E P M$, onde foi considerado significante, dados com $\mathrm{p}<0,05$ (ANOVA de uma ou duas vias, seguido de Teste de Bonferroni).

\section{RESULTADOS E DISCUSSÃO}

$\mathrm{Na}$ etapa de caracterização do modelo, observou-se que os animais apresentaram hiperglicemia $(425,66 \pm 40,34 \mathrm{mg} / \mathrm{dl})$ em relação ao grupo controle $(88,6=5,6 \mathrm{mg} / \mathrm{dl})$, polidipsia $(D M=190,66 \pm 13,34$ $\mathrm{ml} / \mathrm{dia} ; \mathrm{CT}=45 \pm 7,5 \mathrm{ml} / \mathrm{dia})$, polifagia $(\mathrm{DM}=44,49 \pm 2,31 \mathrm{~g} / \mathrm{dia} ; \mathrm{CT}$ $=22,68 \pm 1,18 \mathrm{~g} / \mathrm{dia})$ e poliúria $(\mathrm{DM}=169 \pm 22 \mathrm{ml} / \mathrm{dia} ; \mathrm{CT}=$ $16,61 \pm 5,81 \mathrm{ml} / \mathrm{dia})$.

Os dados obtidos demonstram que o DM altera propriedades do PAC do nervo ciático representado pela Figura 1, onde observa-se no painel $B$ uma redução da amplitude desde sinal descrito a seguir.

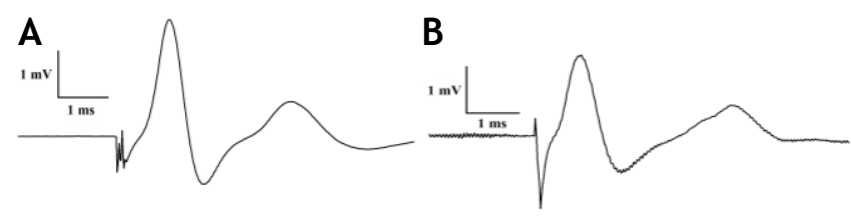

Figura 1. Traçado Representativo do PAC. Painel A representada o traçado do Grupo CT. Painel B representa o traçado do grupo DM. (Fonte: Dados da Pesquisa).

$\mathrm{Na}$ análise da excitabilidade do PAC (Figura 2), observou-se que houve um aumento significativo da reobase no grupo DM $(5,686 \pm 0,49 \mathrm{~V})$, em relação ao grupo CT $(4,167 \pm 0,26 \mathrm{~V})$, correspondendo a um aumento de $36,45 \%$. Não houve alteração da cronaxia (DM = 58,50 $\pm 2,59 \mathrm{~ms} ; \mathrm{CT}=55,75 \pm 4,3$ ).

Entretanto, quando avaliado as propriedades de condutibilidade do PAC (Figura 3), observou-se a diminuição da amplitude da $1^{\text {a }}$ componente no grupo $\mathrm{DM}(1,33 \pm 0,26$ A) em relação ao CT $(2,22 \pm 0,19$ A) que correspondeu a uma redução de $40,09 \%$. Não houveram alterações significativas da Duração e Velocidade das componentes ou da Amplitude Pico-a-pico (APP) do PAC. 
A

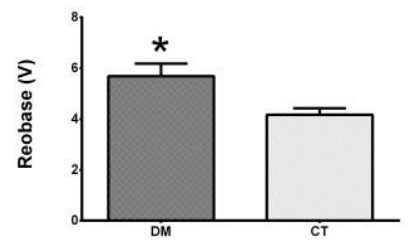

B

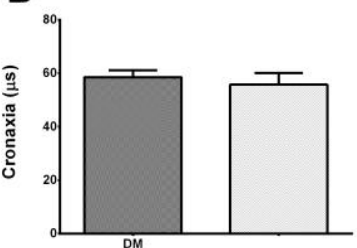

Figura 2. Alteraç̃̃es na Excitabilidade nervosa no DM induzido por STZ.

Dados representados por média \pm EPM (ANOVA de uma via, seguido por pós-teste de Bonferroni, $\mathrm{p}<0,05)$ (Fonte: Dados da Pesquisa).

A

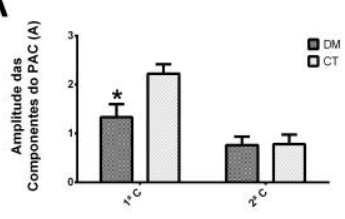

C

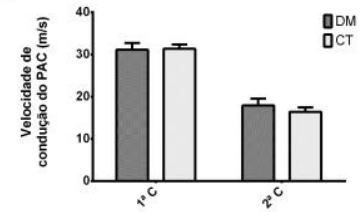

B

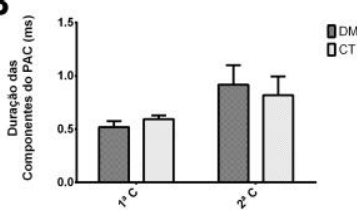

D

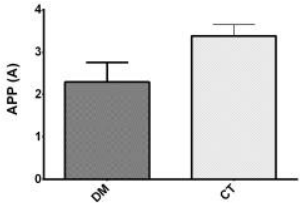

Figura 3. Alterações na condutibilidade nervosa no DM induzido por STZ.

Dados representados por média \pm EPM (ANOVA de uma via, seguido por pós-teste de Bonferroni, $\mathrm{p}<0,05$ ) (Fonte: Dados da Pesquisa).

Em seu trabalho, Lirk e colaboradores (2015) apontam que em modelos de diabetes induzido por dieta, ocorrem alterações na excitabilidade nervosa e associam estas alterações a neurotoxicidade causada pelo quadro de hiperglicemia crônica.

Estudos recentes, apontam que a hiperglicemia decorrente do DM, gera lesões nas células responsáveis pela mielinização do Sistema Nervoso Periférico (SNP), as células de Schwann (FREEMAN et al., 2016). Considerando que a bainha de mielina é responsável por aumentar a velocidade de condução, é esperado que esta patologia leve a alterações da condutibilidade nervosa (LIRK et al., 2015). No entanto, no presente trabalho, a única alteração encontrada nos parâmetros de condutibilidade (Amplitude, Duração e Velocidade das componentes do PAC) foi a queda da Amplitude da $1^{\text {a }}$ Componente, indicando que um menor número de fibras nervosas passou a contribuir para este sinal.

\section{CONCLUSÕES}

No presente estudo, observou-se que o modelo de diabetes adulto induzido por STZ alterou a excitabilidade nervosa por aumentar o limiar do PAC, assim como reduziu a amplitude da $1^{\text {a }}$ Componente do PAC.

\section{REFERÊNCIAS}

FREEMAN, O. J. et al. Metabolic Dysfunction Is Restricted to the Sciatic Nerve in Experimental Diabetic Neuropathy. Diabetes. v. 65, n. 1, p. 228-38, 2016.

LIRK, P. et al. Effects of early and late diabetic neuropathy on sciatic nerve block duration and neurotoxicity in Zucker diabetic fatty rats. Br J Anaesth. v. 114, n. 2, p. 319-26, 2015.

VINIK, A. I. et al. Diabetic neuropathy. Endocrinol Metab Clin North Am. v. 42, n. 4, p. 747-87, 2013. 